\title{
Discussion on the Principle and Reliability Improvement of AC Magnetic Flux Leakage Detection of Steel Rod
}

\author{
Baishang Hu \\ Shaoguan Baosteel Special Steel Co., Ltd., Shaoguan, China \\ Email address: \\ hbsh7750@163.com \\ To cite this article: \\ Baishang Hu. Discussion on the Principle and Reliability Improvement of AC Magnetic Flux Leakage Detection of Steel Rod. American \\ Journal of Physics and Applications. Vol. 7, No. 2, 2019, pp. 48-54. doi: 10.11648/j.ajpa.20190702.13
}

Received: March 13, 2019; Accepted: May 7, 2019; Published: June 4, 2019

\begin{abstract}
In this paper, the principle of ac magnetic flux leakage (referred to as MFL) detection is presented by analyzing the formation of ac magnetic flux leakage and how it is collected. It is found that ac excitation frequency is a very important equipment parameter for ac magnetic flux leakage detection of steel rod. High-frequency current not only stabilizes the penetration depth of ac magnetic field under the rod surface and leakage magnetic field of defects, but also improves the ability to detect defects and adapts to higher detection speed. It is concluded that the lift-off effect is the fundamental reason affecting the reliability of MFL detection of steel rod, and there is an inherent signal amplitude deviation from the irremovable probe bouncing on the surface of steel rod. Then, the factors that aggravate the lift-off effect are analyzed synthetically, and the corresponding countermeasures are put forward. It provides an effective basis for improving the test reliability and reasonably controls the quality risk of the rod.
\end{abstract}

Keywords: Steel Rod, MFL Detection, Principle, Ac Excitation Frequency, Reliability, Lift-Off Effect

\section{Introduction}

The surface finish of hot rolled steel rod is poor, better signal-to-noise ratio (referred to as SNR) and greater detection depth can be obtained by using MFL detection method which is superior to Eddy current detection method [1-3] 126. At present, there are two sets of MFL detection equipment arranged in shaoguan baosteel special steel co., LTD 's large rod detection line and middle rod detection line, and more MFL detection equipment will be put into use one after another. The large diameter rod detection line MFL detection equipment is used to detect steel rods with diameters of $\Phi 70$ $180 \mathrm{~mm}$. The steel rod is driven by a V-shaped roller and determines that the central line is being tested; The magnetic leakage device of the small diameter rod detection line is used to detect steel rods with a diameter of $\Phi 20 \sim 80 \mathrm{~mm}$. The steel rod is driven by a three-claw roller and the central line is determined to detect the steel rod. In order to adapt to the increasing detection tasks, the detection speed has been improved in stages since the equipment was put into use. In each phase of the speed - lifting test, there are problems of detection reliability. The factors affecting the reliability of detection are so numerous and complex that it is difficult to classify and deal with them effectively. In view of this, we began to discuss the principle of MFL detection of steel rods, during which we found that ac magnetization frequency is a very important equipment parameter in MFL detection; Finally, the encouraging result is that we find that the lift-off effect is the root cause that affects the reliability of test signals during detection. Therefore, we comprehensively analyze various factors that aggravate the lift-off effect and put forward corresponding countermeasures to ensure the reliability of testing and effectively control quality risks.

\section{Principle of MFL Detection for Steel Rods}

\subsection{Mechanism of Leakage Magnetic Field Formation}

The workpiece is magnetized by the excitation source (magnetic yoke) during Direct current (referred to as DC) MFL detection. Under ideal conditions, all magnetic field lines pass through the workpiece and almost no magnetic flux leaks to the workpiece surface. Once there is a defect on or below the surface of the workpiece, the magnetic field near the defect is compressed so that the density of the magnetic field 
line at that point increases, then the magnetoresistor near the defect increases, and finally the magnetic field is distorted. As a result, most of the magnetic field lines bypass the defects in the workpiece, and a small part passes through the defects, and some parts leave the workpiece surface to form a leakage magnetic field in the air around the defects [4] 41-42 [5] 31-33 [6], as shown in Figure 1. Leakage magnetic field is detected by magnetic sensor and converted into electrical signal. After amplification and filtering, defect signals and acousto-optic alarm are given, and the defect is finally detected [3] 82-87 [6] [7].

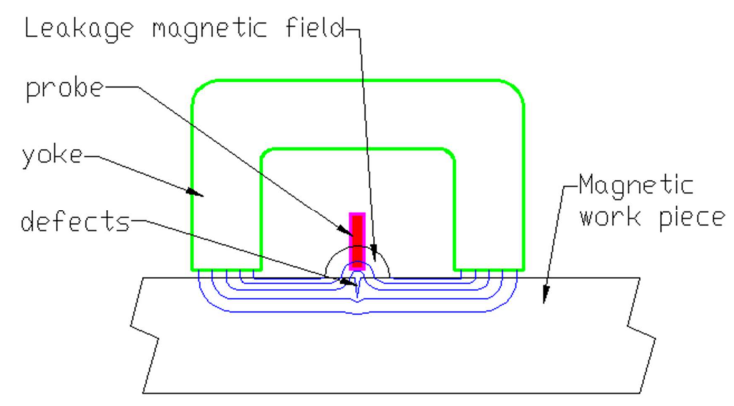

Figure 1. Formation of leakage magnetic field.

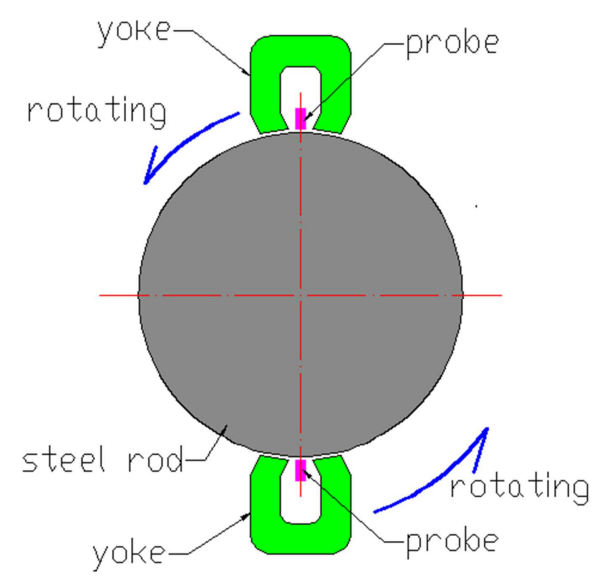

Figure 2. Probe scanning on steel rod surface.

The MFL detection method of steel rod is shown in Figure 2. In the process of detection, the steel rod advances along a straight line, and a pair of magnetic yoke and a pair of probes are set symmetrically on the axis of the steel rod to rotate around the steel rod to realize the spiral sweep of the surface of the steel rod. High-frequency alternating current (referred to as AC) magnetization method is used for MFL detection of steel rods. High-frequency magnetization produces skin effect [8] [9], making most of the magnetic field pass through the surface area of the steel rod. In this way, the surface area of the bar under the magnetic yoke can be saturated magnetized. Once there is a defect in the surface area of the steel rod, part of the magnetic field near the defect is immediately squeezed out of the surface of the rod to form a leakage flux [3] 20-21 [4] $12-13$ [5] 36-37.

\subsection{Acquisition of MFL and Signal Formation}

Coil is used as a sensor in the AC MFL detection of the steel rod, and the signal of the defect is obtained through the electromagnetic induction between the coil and the MFL during the detection process. Usually, The coil is placed perpendicular to the surface of the steel rod and the horizontal component of the defect leakage magnetic field is collected during detection [3] 79-80, 95-96 [7, 10-13]. as shown in Figure 3). If a narrow crack on the surface of the steel rod is detected, the horizontal component $\mathrm{B}_{\mathrm{t}}$ of the spatial distribution of the MFL can be represented by a simple approximate formula (1) [14]:

$$
\mathrm{B}_{\mathrm{t}}=(\mathrm{w} / \pi)\left(\mathrm{y} /\left(\mathrm{x}^{2}+\mathrm{y}^{2}\right)\right) \mathrm{B}_{0} \sin 2 \pi \mathrm{ft}
$$

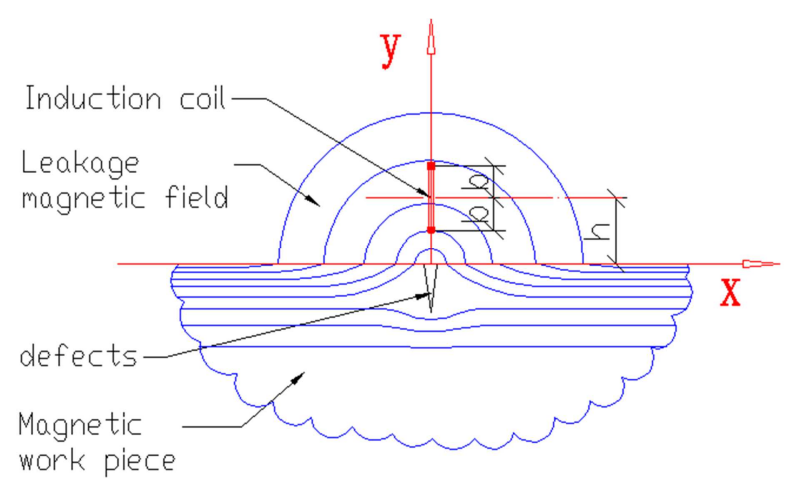

Figure 3. The induction coil scans the leakage field of the defect.

In the formula: $w$ is the longitudinal width of the crack, $\mathrm{m}$; $\mathrm{B}_{0}$ is the flux density at the bottom of the defect, $\mathrm{wb} / \mathrm{m}^{2} ; \mathrm{x}$ is the horizontal distance from a point in the MFL to the center of the defect, $\mathrm{m}$; $\mathrm{y}$ is the vertical distance from a point in the MFL to the center of the defect, $\mathrm{m}$; $\mathrm{f}$ is the $\mathrm{AC}$ frequency, $\mathrm{Hz}$; $\mathrm{t}$ for the time, $\mathrm{s}$.

In general, There are at least 10 sinusoidal signals contained in the modulation signal of the AC MFL [3] 126 [15, 16] (this determines the upper limit of the sweep speed of the AC MFL detection), That is, the defect sweep time that forms the detection signal is at least one order of magnitude larger than a sinusoidal alternating cycle. Therefore, the change in coil magnetic flux caused by the sweep speed is negligible compared to the change in magnetic flux in the collar caused by the high-frequency alternating magnetic flux. Assume that the width of the coil is $2 b$, the length of the coil (the length entering the paper) is 1 , the number of coil turns is $\mathrm{N}$, and the distance from the center of the coil to the surface of the steel rod is $\mathrm{h}$ (called the distance of the coil). Taking the Bt value at the center of the coil as the average of the leakage flux density through the entire coil, the inductive voltage of the coil can be calculated as formula (2) according to Faraday law of electromagnetic induction [3] 74 and formula (1):

$$
\mathrm{V}=-\mathrm{N}(\mathrm{d} \varphi / \mathrm{dt})=4 \mathrm{wlNfB}_{0}\left(\mathrm{bh} /\left(\mathrm{x}^{2}+\mathrm{h}^{2}\right)\right)
$$

In the formula: $h$ is the distance from the center of the coil to the surface of the workpiece, $\mathrm{m}$; $\mathrm{b}$ is half coil width, $\mathrm{m}$; 1 is the coil length, m;

From formula (2), it can be seen that when the distance value of $h$ does not change, the inductive voltage in the coil depends on the position $\mathrm{x}$ of the coil in the MFL, and when 
$\mathrm{x}=0$, the inductive voltage reaches a maximum. During the test, the coil is continuously spiral along the surface of the steel rod. Once there is an axial crack on the surface area of the steel rod, the coil can certainly reach $\mathrm{x}=0$ points to obtain the highest inductive voltage.

It can also be seen from formula(2) that the high frequency of the AC magnetic field has the following effects: When the other factors in formula (2) are certain, The high frequency of AC magnetization can not only stabilize the skin depth of the magnetic field in the steel rod (the skin depth is stable at about $1 \mathrm{~mm}$ when the frequency is higher than $1000 \mathrm{~Hz}$ [3] 124 [16] [17]), but also effectively improve the induction voltage from the MFL and improve the SNR. At the same time, higher ac frequency is conducive to better formation of modulation signal. In other words, defects of smaller width MFL can be detected. Correspondingly, detection speed can be improved to detect defects of certain size. However, it is not the higher the better for ac magnetization frequency, due to the MFL signal frequency is equal to the frequency of the ac MFL and ac excitation frequency, we should comprehensively consider the power of yoke magnetization and the performance of the magnetic core and hysteresis characteristics of steel rod, as well as the size of the defect was detected in the rod rolling surface area, etc., in general, magnetizing current is designed in the $1 \sim 10 \mathrm{KHz}$ [5] 126

In addition, for a certain detection situation, $\mathrm{w}, \mathrm{l}, \mathrm{N}, \mathrm{f}$, and $\mathrm{B}_{0}$ in formula (2) are all constants. Therefore, the maximum value of coil induction voltage can be simplified as:

$$
\mathrm{V}=\mathrm{K} /(\mathrm{h} / \mathrm{b})
$$

In the formula: $\mathrm{K}=4 \mathrm{wlNfB}_{0}$;

The ratio of $\mathrm{h} / \mathrm{b}$ is called normalized lift-off value, referred to as lift-off value. In the process of detection, the change of $\mathrm{h} / \mathrm{b}$ will produce the lift-off effect and cause the amplitude of MFL signal to fluctuate.

It can be seen from formula (3) that the maximum induced voltage of the coil is inversely proportional to the lift-off value of the coil for the MFLof the identified defect. The detection signal of the coil corresponds to different lift-off value $\mathrm{h} / \mathrm{b}$, as shown in Figure4. This confirms the statement "... When the lift-off value exceeds twice the crack width, the strength of the MFL decreases rapidly with the increase of the lift height" [3]. In terms of the $h / b$ value versus the signal amplitude. MFL signal amplitude increases with the decrease of the values of $\mathrm{h} / \mathrm{b}$ in figure 4 , the ideal maximum is the lower side of the coil on the rod surface, the $h / b=1$, but in the actual detection, induction coil encapsulation in wear-resistant boot (see figure 5), the distance between the induction coil and the rod surface is not less than the thickness of wear-resistant pieces, this limits the minimum value of $h / b$, in the process of testing for a long time, as wear-resistant pieces of thinning the minimum value for $h / b$ becomes smaller, and higher detection sensitivity, but the noise will also rise to lower SNR, wear-resistant boot need to be replaced when SNR is not meet the requirements.

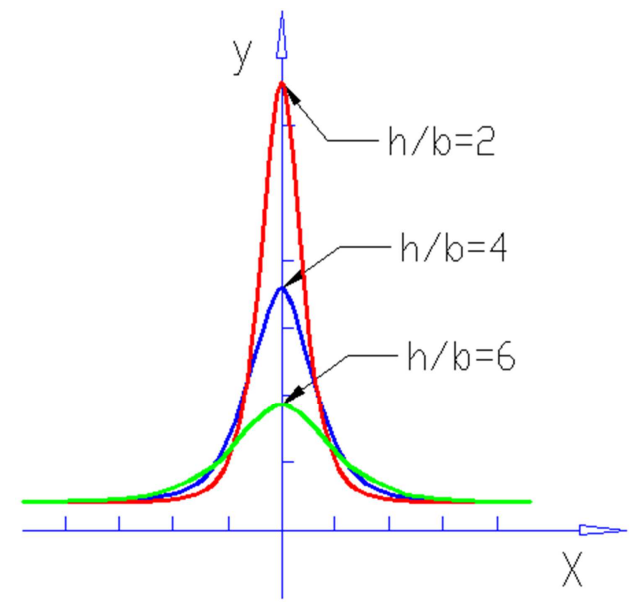

Figure 4. Effect of lift-off effect on the detection signal.

As for the setting of the induction coil, In the actual detection, a set of coils is often arranged on each side of the probe substrate to obtain higher sensitivity and SNR, and the two sets of coils are connected in a differential manner to form a probe channel. The modulated signal obtained from the coil channel is demodulated and displayed in the same direction by the bridge rectifier. Generally, the length of a single coil should not exceed $30 \mathrm{~mm}$ to ensure sensitivity and SNR. On the other hand, multi-channel coils are often arranged side by side to increase the scanning width and improve the scanning efficiency [3] 80 [6,12,13,15], but limited by the length of the magnetic yoke, the length of the coils side by side must not exceed the length of the magnetic yoke.

\section{Analysis of the Factors Affecting the Reliability of MFL Detection of Steel Rod and Countermeasures}

\subsection{Surface Roughness of Steel Rods}

The probe consists of a wear-resistant boot and an induction coil inside. Ideally (the surface of the steel rod is smooth and has no longitudinal bending, and the probe is subjected to a uniform push pressure from the spring). The wear-resistant boot slides against the surface of the steel rod during detection. At this time, The coil is kept constant relative to the lift-off value $\mathrm{h} / \mathrm{b}$ on the surface of the steel rod. When the detection parameters are unchanged, the same defect on the steel rod is repeatedly scanned and the signal amplitude formed is consistent. However, in actual testing, the probe is subjected to the combined effect of spring pressure, rotating centrifugal force, and support force on the rough surface of the steel rod. The wear-resistant boot bounds quickly on the surface of the steel rod and makes a clatter sound, as shown in Figure 5. The induction coil bounce with the wear-resisting boot, and the lift-off value $(\mathrm{h} / \mathrm{b})$ is difficult to be constant when the induction coil is scanning the defect. The test result is that the detection signal value obtained from each scan is changed from the same defect. This creates uncertainty that the defect be effectively detected. The bounce of induction coil also 
brings vibration noise signal, so that the detection SNR is reduced.

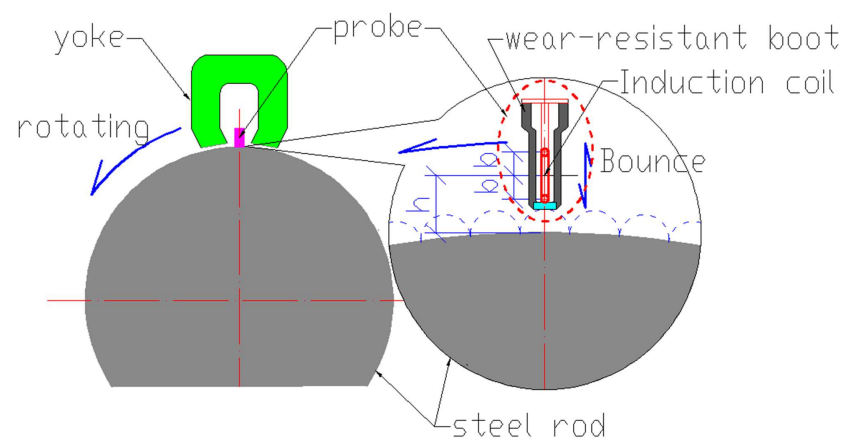

Figure 5. Probe bouncing in the process of scanning.

Once a violent probe bounce occurs during the MFL detection of the steel rod, the defect may be missed or misreported, even if the conveyor rollers and the device have a good degree of consistency, and the straightness and roundness of the steel rod are in full conformity with the standard. It is difficult to eliminate the probe bounce in the detection process. The main reasons have been described before. Therefore, the deviation of the detection results caused by the probe bouncing is often referred to as the inherent deviation of the device. The characteristic of this inherent deviation is that the signal amplitude fluctuates within a certain range, and the range of this fluctuation depends on the amplitude of the probe bounce. It is easy to cause false alarm or missed inspection when the reference artificial injury signal is just past the alarm gate, but it will not cause a large defect missed inspection. In general, the appropriate sensitivity compensation is added to the verification sensitivity before the test is carried out to eliminate the risk of defects being missed.

The amplitude and span of the probe's bouncing are related to the rotation speed of the probe. They increase with the increase of the rotation speed, resulting in the increase of the lift-off effect, which shows that the accuracy and stability of the detection are poor. The rotation speed of the probe depends on the diameter of the steel rod and the detection speed in ensuring that the steel rod surface is fully covered. In addition, the scanning speed of the probe on the surface of the steel rod should not be too fast. Therefore, testing procedures should be developed for different diameter steel rods to overcome indirect effects of scanning speed. For example, care should be taken to reduce the detection speed appropriately for large diameter steel rods.

\subsection{The Concentric Degree of Equipment}

The concentric degree here refers to the coaxial degree of the center of the probe rotation and the center axis of the forward steel rod. The reliability of MFL detection is seriously affected by the concentric degree of the equipment. Figure $6 \mathrm{a}$ shows the scanning signal with uniform circumferential sensitivity, (In Figure 6, the horizontal direction indicates the direction of the length of the sample bar, the vertical direction indicates the amplitude of the scanning signal, and the regional color difference represents the range of the amplitude used to evaluate the severity of the signal. Figures 8 and 9 are similar to this figure). There are many factors that affect the coaxiality. First, there are inconsistencies in the positioning of the center of rotation of the probe and the axis of the steel rod center by the transmission roller, which make the difference of circumferential sensitivity too large, as shown in Figure $6 \mathrm{~b}$, At this time, It is easy to cause false alarm on the side of the probe close to the steel rod, and it is easy to cause leakage on the side of the probe far from the steel rod. The second is that the linear path of the roller channel is not good, which causes the steel rod to beat when it is transmitted, thus causing the instantaneous lift-off effect of the probe, and the instantaneous lift-off effect increases with the deterioration of the transmission rollers path linear and the increase of the transmission speed. There is an increased chance of missing and misreporting. The third is that the centring roller and the transmission V-type roller before and after the host are worn after the equipment has been running for a long time, resulting in the deterioration of the coaxial degree of the equipment and the shaking of the steel rod in the transmission.

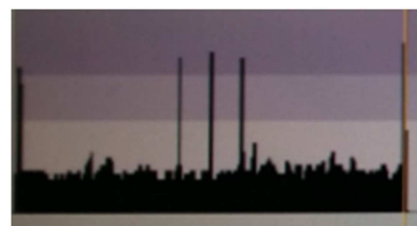

(a)

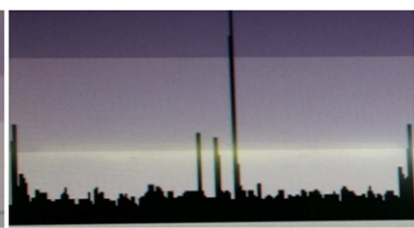

(b)
Figure 6. The difference of circumferential direction signal caused by the deterioration of the concentric degree.

Specific measures to repair the equipment are taken to improve the coaxiality of the equipment and the transmission rollers based on the reasons described above. For example, the coaxiality of the roller channel relative to the center of the probe rotation is regularly adjusted, and the linear line of the roller channel transmission path is regularly calibrated. The wear condition of conveying and axial setting roller is checked in due course, and the badly worn roller is replaced in time.

\subsection{Symmetry of Rotating Probes}

When the support of the magnetic yoke is asymmetric relative to the rotating center on both sides, the pressure of the wear-resisting boots on the surface of the steel rod is inconsistent during the detection process, which increases the difference in the bouncing amplitude of the probes on both sides. This increases the difference in sensitivity between the two probes. At the same time, The difference increases with the speed of scanning. A similar result comes from the difference in thrust between the two probe springs. This will seriously affect the stability of equipment calibration.

The way to overcome this is to adjust the magnetic yoke position to reduce the symmetry deviation between two sides, and check to confirm that the spring thrust of the two probes to be the same. The position of the two magnetic yoke support in the rotating body of the magnetic leakage machine located in 
the large diameter rod detection line can be adjusted separately, and it is easy to correct the asymmetry of the probe. The position of the two magnetic yoke support in the rotating body of the magnetic leakage machine located in the small diameter rod detection line must be synchronously adjusted. It is necessary to cooperate with the roller to be adjusted to eliminate asymmetry between magnetic yoke supports 。

Normally, the wear-resistant boot is parallel to the axis of rotation and points vertically to the center of rotation, but if the wear-resistant boot is not assembled or operated in accordance with the above requirements, such as the wear-resistant boot deflection or tilt relative to the axis of rotation. This leads to uneven lift values and inconsistent sensitivity of the coils on both sides of the probe, as well as differences in sensitivity between different coil channels in a set of probes. As shown in Figure 7, it can be seen from the wear condition of wear-resistant boots that Figure 7 a shows the normal wear state, while Figure $7 \mathrm{~b}$ shows the abnormal wear state.

The countermeasures for the above anomalies are to check the wear condition of wear-resistant boots frequently, adjust the position accuracy of wear-resistant boots according to the sensitivity difference of each channel, and conduct static balance calibration for all coil channels of the probe.

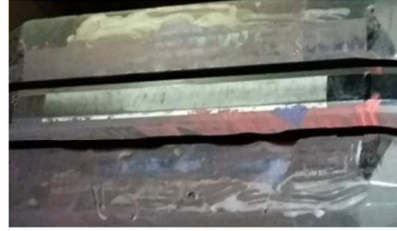

(a)

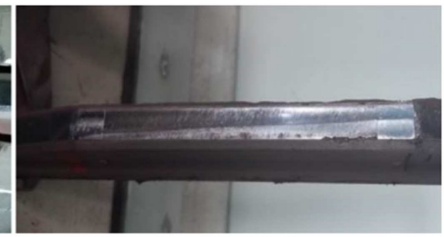

(b)
Figure 7. Wear of wear - resistant boots

\subsection{Bending of Steel Rods}

The bending of the steel rod includes the bending of the head and/or tail and the bending of the central axis. Coaxial deviation occurs when the local bending part of the steel rod passes through the rotating body of the MFL detection equipment. The detection sensitivity of the convex side of the steel rod is on the high side, and the possibility of false alarm is large, while that of the concave side is on the contrary. If there is bending of the head and tail of the steel rod, the impact will be greater, because the head and tail bending will not only lead to false alarm signal (as shown in Figure 8), but also impact the three-claw roll to loosen or damagedue to the eccentric entry and exit of the steel rod. As a result, the unsteady clamping of the three-jaw roller on the the steel rod, the vibration of the steel rod intensified. At the same time, excessive bending of the steel rod head and tail may also damage the rotating wear-resistant boots. The solution to this problem is to install a strict "watchdog" guide in front of the device to limit over-bent steel rods entering the device and tighten the three-claw rolls before and after the device to reduce the vibration of the steel rods appropriately.

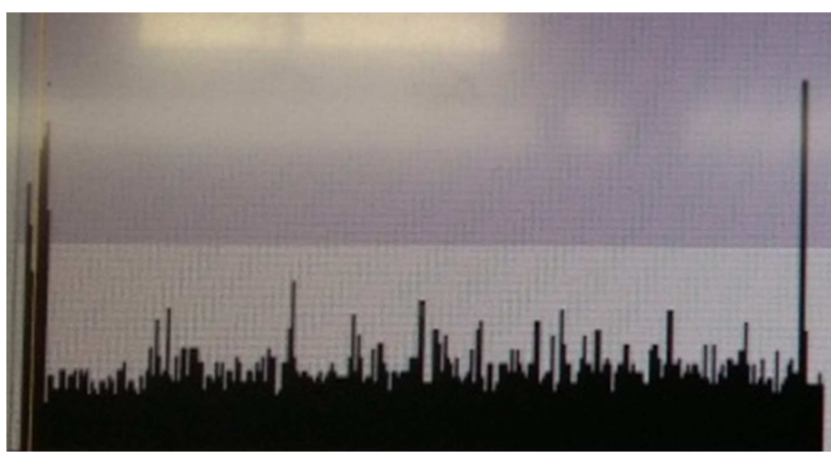

Figure 8. Alarm signal caused by the bending of the head and tail of the steel rod.

When a small diameter steel rod (usually less than $\Phi 30 \mathrm{~mm}$ ) is detected, it is easy to produce a head and tail swing due to the lack of rigidity. When the three-jaw roller is loose, the swing of the steel rod is more serious, because when the head of the steel rod enters into the detection device, only the three-jaw roller at the entrance holds the steel rod, and the roller at the exit is not working. Similarly, only the three-jaw roller at the exit holds the steel rod, and the roller at the entrance has been separated when the end of the steel rod leaves the detection device. At these times, the steel rod head and tail easily free swing, resulting in missed or false alarm. Overcome method is to install a entry - guided sleeve (less than $\Phi 28 \mathrm{~mm}$ ) and tighten the three - roll claw in the sleeve.

\subsection{Oxides on the Surface of Steel Rods}

The distribution of the MFL around the probe changes when Oxide adsorption on the surface of the steel rod or the surface of the wear-resistant boots, thus the flux through the probe coil also changes. The effect is similar to the change of the coil lifting off, In general, the oxide skin is easily left on the surface of the rough steel rod, and iron oxide powder is easily attached to the pits and grooves on the surface of the rough steel rod. Dust and oxide powder are easily attached to the magnetic yoke and wear-resistant boots in the following situations: the vibration of the steel rod during transmission, the clamping of the three-claw roller before and after the host, the steel rod surface being hit by wear-resistant boots, and the magnetic field exists between the magnetic yoke and the steel rod. The MFL is mainly caused by the rough surface of the steel rod, and the oxidized powder with high magnetic conductivity is easily absorbed between the magnetic yoke and the wear-resistant boots (as shown in Figure 9a) or enters into the crack of the damaged wear-resistant boots (as shown in Figure9b) to form a alternating magnetic poles, resulting in disorderly noise, poor SNR, and even serious false alarm, as shown in Figure 9c.(In Figure 9c, the horizontal position of the small points distributed in the lower half corresponds to the axis position and length of the defects on the bar, and the longitudinal position represents the circumferential expansion position corresponding to the orientation of the steel rod section where the defects are located. The yellow line is the timely coordinate line of the manual click signal) Because the concentrated oxide powder has a polymerizing effect on the 
weak magnetic field around the wear-resistant boots [3] 79 [6]. The countermeasure of the above problems is to improve the surface finish of steel rod during straightening. Compressed air and electric brush are used to remove residual oxides on the surface of the steel rod. Purge and clean the detection device timely; Discover and replace damaged wear - resistant boots in time.

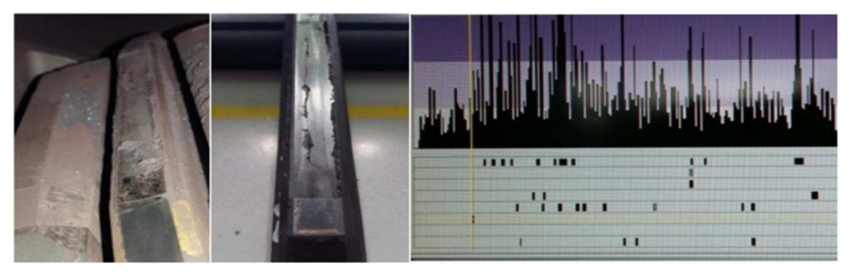

(a)

(b)

(c)

Figure 9. The condition of wear-resistant boots and false alarm caused by the oxidation and powder.

\section{Conclusion}

1) In this paper, the Principle of ac MFL detection of bar is given, and it is found that ac excitation frequency is a very important equipment parameter for MFL detection of steel rod. High-frequency excitation current produces high-frequency alternating magnetic field. This results in the skin effect of the magnetic field in the steel rod, so that the surface area under the magnetic yoke is magnetized to saturation, which is conducive to the formation of a stable MFL corresponding to the defect. At the same time, The high frequency of MFL effectively improve the induced voltage of the coil sweeping over the leakage magnetic field, as well as improve the SNR and the ability to detect defects. The high-frequency ac excitation current is conducive to detecting the MFL with a smaller width corresponding to the defect, so that the identified defect can be detected at a higher speed.

2) There is an inherent deviation of signal amplitude in the dynamic MFL detection of rod, which is caused by the dynamic lift-off effect of probe and rod surface that can not be eliminated.

3) Lift-off effect is the most serious factor affecting the reliability of AC MFL detection of steel rods. The factors that cause the lift-off effect and its dynamic differences may be rough surface of the steel rod, poor concentric degree of equipment and conveying roller and clamping roller, asymmetry of the rotating probe (including asymmetric distance and asymmetric position), difference in thrust of the spring, and bending degree of the steel rod, etc.. There is also a similar effect of surface adhesion oxides. In the actual detection, these factors often exist in association, so that the reliability of the test results are affected by multiple comprehensive effects. Measures are taken to minimize the negative impact on test reliability so as to maximize the function of MFL detection equipment by analyzing the comprehensive impact factors in the testing process. This is of great significance to ensure the quality of steel rods delivered.

\section{References}

[1] DENG Zhi-yang, YANG Yun, FENG Bo, KANG Yi-hua. Influence of Surface Roughness on MFL Inspection of Cracks [J] Non-destructive testing. 2016 No. 02 pp. 40-44.

[2] TANG Qi, FENG Bo YANG Yun KANG Yi-hua. Influence of Surface Roughnesson Eddy Current Testing [J]. Non-destructive testing. Issue No. 03 2016. Pp. 47-51.

[3] Fanhong. Magnetic leakage detection of ferromagnetic metal materials $[\mathrm{M}]$. Committee for the Accreditation and Certification of Metallurgical Non-destructive Test Personne.. Beijing: China Science and Technology Press. 2016.

[4] Songzhizhe. Magnetic powder testing (2nd edition) [M]. Organized by the China Special Equipment Inspection Association, Beijing: China Labor Social Security Press. April 2007, No. 2.

[5] Yedaiping and Suliguang. Magnetic powder testing [M]. Committee on the Compilation and Examination of Training Materials for Qualification and Certification of Non-destructive Testing Personnel in National Defense Science and Technology. Beijing: China Machine Press. March 2004.

[6] LIN Jun-ming. Research on Magnetic Flux Leakage Detection Technology and Its Development Status. [J] Non-destructive testing. Vol. 30 No. 1 February. 2006: 1 5.

[7] Lijiawei. Non-destructive testing manual (2nd edition) [M]. Beijing: China Machine Press. 2011. pp. 5-31 5-34.

[8] Xu Yanfang. Qualitative explanation of skin effects [J]. Journal of Teachers College of Qingdao University. No. 2, 1997. pp. 72-73.

[9] Huang Changming. Analysis and discussion on skin effect [J]. J. of Henan Univ (Nat.Sci.). Vol. 23, No. 3, 1993. pp. 46-47.

[10] Kang Yihua Wu Xinjun Yang Shuzi. SIGNAL PROCESSING TECHNOLOGY FOR MAGNETIC [J] Non-destructive testing. Vo 1. 22 No. 6 June 20 0:255 249.

[11] DU Zhi-Ye1, RUAN Jiang-Jun, YU Shi-Feng, Selection of Radial and Axial Components from Magnetic Flux Leakage Signals [J]. Non-destructive testing. Vol. 32, No. 07 2010: 480 484

[12] LIAO Changrong, LIAO Zheng, HAN Liang, WANG Binbo, SHI Xiangcong, XIE Yunshan. Nalytical study on crack magnetic flux leakage for ferromagnetic componentand detection system based on geomagnetic field [J]. Journal of Chongqing University. Vol. 35 No. 10 Oct. 2012: 77 84.

[13] HAN Ling, LIAO Chang-rong, WANG Bin-bo, SHI Xiang-cong, XIE Yun-shan, WEI Wen-xiong. alytical Study on Crack Magnetic Flux Leakage for Ferromagnetic Components and It's Detection Device [J]. Journal of Southwest University (Natural Science Edition) Vol. 34 No. 7 Jul. 2012: 1 6.

[14] American Society of Nondestructive Testing (ASNT). Nondestructive Testing Handbook-Magnetic powder Testing [M]. Translation and Review Committee of American Society of Nondestructive Testing. Shanghai: World Book Inc. 1992, pp. 259-265.

[15] GB/T32547-2016. Method for magnetic flux leakage testing of round steel $[\mathrm{S}]$. 
[16] LI Luming, HUANG Songling, SHI Keren. AC and DC magnetizing for magnetic flux leakage testing [J]. J Tsingh ua Univ (Sci \& Tech), 2002, Vo 1. 42, No. 2.
[17] FAN Hong, JIA Hui-ming. Exploration on the steel rods AC magnetic flux leakage testing technique [J]. Physics Examination and Testing. Vol. 33, No. 6, 2015. pp. 1-5. 